\title{
Estudio de la anteversión femoral
}

\author{
CASTELEIRO GONZÁLEZ, R.*; GÓMEZ PELLICO, L.**; FERNÁNDEZ-VALENCIA, R.**; \\ CASTAÑO ZAPATERO, C** y FERNÁNDEZ CAMĀCHO, F.J.** \\ Servicio de Traumatología y Cirugía Ortopédica. Hospital General Greogorio Marañón * \\ Departamento de Ciencias Morfológicas y Cirugía. Facultad de Medicina. Universidad de Alcalà de Henares. **
}

\section{RESUMEN}

Se estudian los valores angulares de la anteversión femoral en 30 fémures humanos secos normales, del lado izquierdo y pertenecientes a individuos adultos.

Los valores angulares de la muestra son sometidos a estudio estadístico dando una fiabilidad de la media para $\mathrm{p}=0,01$.

Se revisa la bibliografía de otras mediciones realizadas en hueso seco y se comparan con los valores obtenidos en este trabajo.

Los valores obtenidos contribuirán a un mejor conocimiento del ángulo de anteversión femoral y consecuentemente a la aplicación clínica del mismo.

Palabras clave: Fémur, anteversión femoral, ángulo de anteversión.

\section{SUMMARY :}

We study the angular values of the femoral anteversion in 30 normal dry human femurs, of the left hand side and belonging to adult individuals.

The angular values of the pattern are put under statistic study giving a liability of the mean for $p=0,01$.

Other patterns bibliography are reviewed done on dry bones and are compared with the values obtained in this study.

The values obtained will contribute to a better knowledge of the femoral anteversion angleand consequently to its clinical application.

Key words: Femur, femoral antversion, anteversion angle.

\section{INTRODUCCIÓN}

El ángulo de anteversión femoral (AAF) viene definido entre las intersecciones del eje cérvico-cefálico y la tangente posterior bicondílea que pasa por el plano horizontal, orientándose en el espacio hacia dentro y hacia delante (fig. 1).

El AAF ha recibido las siguientes denominaciones: ángulo de rotación ${ }^{1}$, ángulo de torsión o giro ${ }^{9}$, ángulo de declinación ${ }^{5}$, etc.
La importancia de este ángulo tanto en su aspecto morfológico como en su aplicación clínica se desprende del número de publicaciones y autores relacionados con su estudio. Para su medida se han empleado diferentes métodos y técnicas, mediciones fluoroscópicas, radiológicas, TAC, ECO, RMN y por último mediciones realizadas en fémur humano seco, destacando las efectuadas por $^{2,4,8,12,14,15,16,17}$ (Tabla 1).

En el presente trabajo se exponen los valores estadísticos del AAF correspondiente a población adulta española, para lo cual se han efectuado mediciones en hueso seco. El conocimiento de estos datos nos permitirá una correcta aplicación clínica del AAF, y así mismo servirán como referencia y elementos de comprobación a los valores obtenidos con otras técnicas de medición.

\section{MATERIAL Y MÉTODO}

Se ha medido el AAF en 30 fémures humanos secos pertenecientes al lado izquierdo de individuos adultos de ambos sexos procedentes de la osteoteca de la Facultad de Medicina de la Universidad de Alcalá de Henares. Fueron descartados los fémures que presentaron deterioro en su conservación o cualquier otra alteración morfológica que pudiera inducir a error al realizar las mediciones.

En cada fémur fue calculado el centro geométrico del cuello femoral (CC), situándose éste en la parte más estrecha del mismo; para calcular este punto se realiza mediciones con calibrador en los diámetros transversales del cuello y calculando para cada diámetro su punto medio.

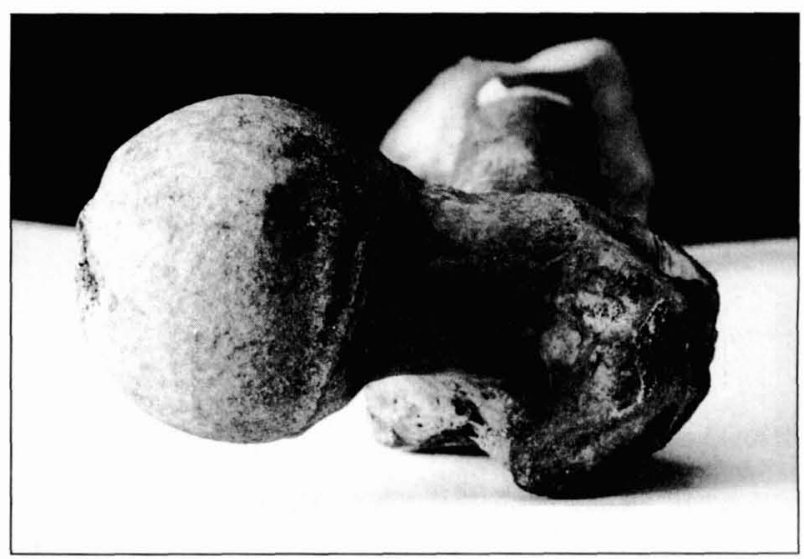

Figura 1. Extremidad superior del f $[$ emur en vista superior en donde se aprecia la orientaci[on de la anteversi[on femoral. 
TABLA 1

REVISIÓN BIBLIOGRÁFICA DEL VALOR DEL AAF EN HUESO SECO DE INDIVIDUOS ADULTOS

\begin{tabular}{|c|c|c|c|c|c|c|}
\hline & Autor & Año & N. ${ }^{\circ}$ Femures & Media & Rango & Notas \\
\hline (12) & Mikulicz & 1878 & & $11,6^{\circ}$ & -25 a 37 & \\
\hline (2) & Broca & 1896 & & $17^{\circ}$ & 2 a 38 & Franceses blancos \\
\hline (2) & Broca & 1896 & & $20^{\circ}$ & & Negros \\
\hline (14) & Parsons & 1915 & 300 & $15,5^{\circ}$ & -17 a 40 & \\
\hline$(4)$ & Durhan & 1915 & 200 & $11,9^{\circ}$ & 0 a 35 & \\
\hline (15) & Pearsons & 1919 & & $15,3^{\circ}$ & & \\
\hline (16) & Pick & 1941 & 152 & $14^{\circ}$ & -18 a 14 & \\
\hline (8) & Kingsley & 1948 & 630 & $7,7^{\circ}$ & -27 a 38 & Fémur izquierdo \\
\hline$(8)$ & Kingsley & 1948 & 630 & $8,5^{\circ}$ & -20 a 33,5 & Fémur derecho \\
\hline (17) & Ruff & 1983 & & $22,7^{\circ}$ & & Indios Pecos S. XVI. \\
\hline
\end{tabular}

Cada hueso fue colocado en la tabla osteométrica (plano horizontal) orientándose en proyección $0^{\circ}-180^{\circ}$ con relación a la cámara fotográfica situada sobre trípode estático. En cada hueso se realizaron tres fotografías de la extremidad proximal en vista superior, que incluye: cabeza femoral, borde superior del cuello y borde superior del trocánter mayor. En cada fotografía fue calculado el centro de rotación de la cabeza femoral (CR), para lo cual la cabeza femoral fue inscrita en un cuadrilátero de lados tangentes a la cabeza, paralelos y perpendiculares con relación al plano horizontal, las bisectrices del cuadrilátero nos determinan el punto CR (fig. 2).

Conocidos los puntos CR y CC trazamos por los mismos el eje cérvico-cefálico (ECC) que con la intersección del plano horizontal (tabla osteométrica) nos permite trazar el AAF en cada fémur. Mediante goniómetro hallamos el valor angular del mismo. Cada una de estas mediciones se repitió por tres veces y en tres momentos diferentes.

Los ángulos obtenidos son analizados estadísticamente estudiándose los valores de dispersión y concentración de la muestra.

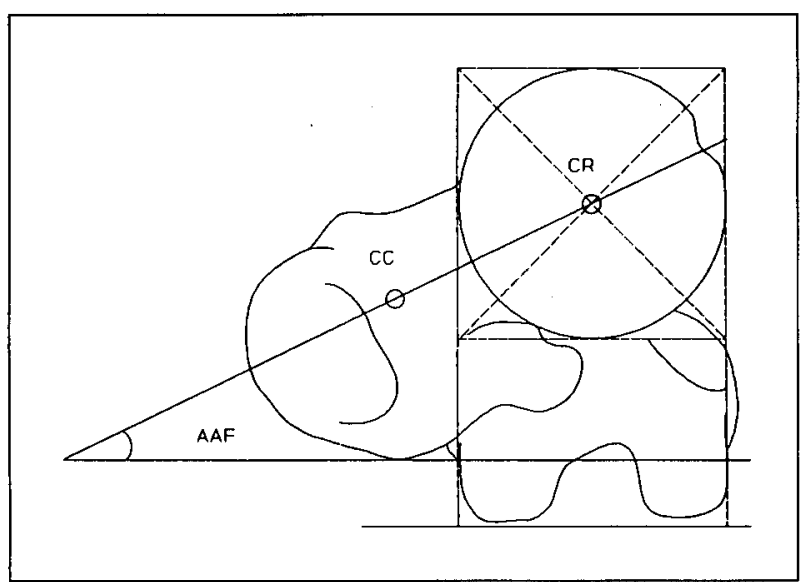

Figura 2. Esquema de la extremidad proximal del f [emur en vista superior donde se refleja la construcci[on geom[etrica para el c[alculo del CR, CC, eje cervicocef[alico e intersecci[on con el plano horizontal para calcular el AAF.
TABLA 2

VALORES ESTADÍSTICOS DEL AAF EN POBLACIÓN ADULTA ESPAÑOLA

\begin{tabular}{cccc}
\hline Fémur & Anteversión & Fémur & Anteversión \\
\hline 1 & $3^{\circ}$ & 16 & $8^{\circ}$ \\
2 & $7^{\circ}$ & 17 & $34^{\circ}$ \\
3 & $6^{\circ}$ & 18 & $3^{\circ}$ \\
4 & $11^{\circ}$ & 19 & $8^{\circ}$ \\
5 & $15^{\circ}$ & 20 & $26^{\circ}$ \\
6 & $5^{\circ}$ & 21 & $16^{\circ}$ \\
7 & $2^{\circ}$ & 22 & $13^{\circ}$ \\
8 & $19^{\circ}$ & 23 & $16^{\circ}$ \\
9 & $16^{\circ}$ & 24 & $23^{\circ}$ \\
10 & $3^{\circ}$ & 25 & $7^{\circ}$ \\
11 & $11^{\circ}$ & 26 & $15^{\circ}$ \\
12 & $2^{\circ}$ & 27 & $6^{\circ}$ \\
13 & $13^{\circ}$ & 28 & $9^{\circ}$ \\
14 & $11^{\circ}$ & 29 & $10^{\circ}$ \\
15 & $0^{\circ}$ & 30 & $13^{\circ}$ \\
\hline
\end{tabular}

\section{RESULTADOS}

Quedan reflejados en la tabla 2 y su distribución de frecuencias en la figura 3.

\section{DISCUSIÓN}

A partir de embriones de $27 \mathrm{~mm}$, correspondientes a 25 días, horizonte $\mathrm{XII}^{13}$ es posible identificar el cuello femoral y medir el AAF, por el contrario, para otros autores ${ }^{9}$, durante el $2^{\circ}$ a $3^{\circ}$ mes del desarrollo embrionario no existe ángulo de anteversión y hasta el $5^{\circ}-6^{\circ}$ mes no es posible medir su valor. Al final del periodo fetal se completa el desarrollo del valor del AAF, permaneciendo estacionario hasta el $2^{\circ}$ a $4^{\circ}$ año de la vida, en que comienza a disminuir para estabilizarse en la edad adulta, si bien los valores angulares decrecen con la vejez. ${ }^{8,10}$

De las mediciones efectuadas por diferentes autores $^{3,6,7,7,9,11}$ podemos concluir que en el periodo embrionario predomina la retroversión y ésta alcanza un rango 
muy amplio, durante el periodo fetal va disminuyendo la retroversión y empieza a desarrollarse anteversión, pudiendo alcanzar valores de $30^{\circ}$ a $50^{\circ}$.

A partir del nacimiento el valor del AAF, va decreciendo para estabilizarse entre los 16 a 18 años ${ }^{18}$, desde esa edad va a permanecer fijo durante toda la vida adulta, de nuevo con la vejez se va a producir una pérdida del valor angular. ${ }^{8,10}$

Referente al sexo, según ${ }^{8}$, no existen variaciones del valor del AAF entre hombres y mujeres, y estos mismos autores han encontrado un valor de aproximadamente $1^{\circ}$ mayor en el fémur derecho sobre el AAF del fémur izquierdo.

A la vista de las variaciones que sufre el AAF se desprende su papel activo como elemento de expresión dinámico de la morfología femoral y su papel en la estabilización de la articulación coxo-femoral, así en los periodos fetales al AAF estabiliza ante la flexión y abducción de la cadera ${ }^{6}$, al iniciarse la bipedestación para que la cadera no se subluxe hacia delante es preciso que el AAF disminuya. Durante esta época existen una serie de movimientos el la articulación de la cadera que debido a la plasticidad ósea van a incidir en el valor de la anterversión, tal es el caso de los movimientos de rotación externa que favorece la retroversión y los movimientos de rotación interna que favorecen los de anteversión ${ }^{6}$. Pero no sólo estos factores van a influir en el valor del AAF, existen otras acciones como la contracción del psoas iliaco que al limitar la rotación externa actúan sobre la retroversión y la musculatura adductora que con su acción varizante tiende a favorecer la anteversión y por último la cápsula articular y los ligamentos capsulares que al limitar la separación de la cadera limitan la retroversión. La acción conjunta de todos los factores reseñados controla el desarrollo del valor del AAF, estabilizándose su valor en la edad adulta.

Las mediciones realizadas en este trabajo coinciden con los datos de Mikulicz ${ }^{12}$ y Durhan ${ }^{4}$, son sensiblemente inferiores a las mediciones de Broca ${ }^{2}$, Pearson ${ }^{15}$, Pick $^{16}$ y Ruff ${ }^{17}$, empleando este autor fémures secos

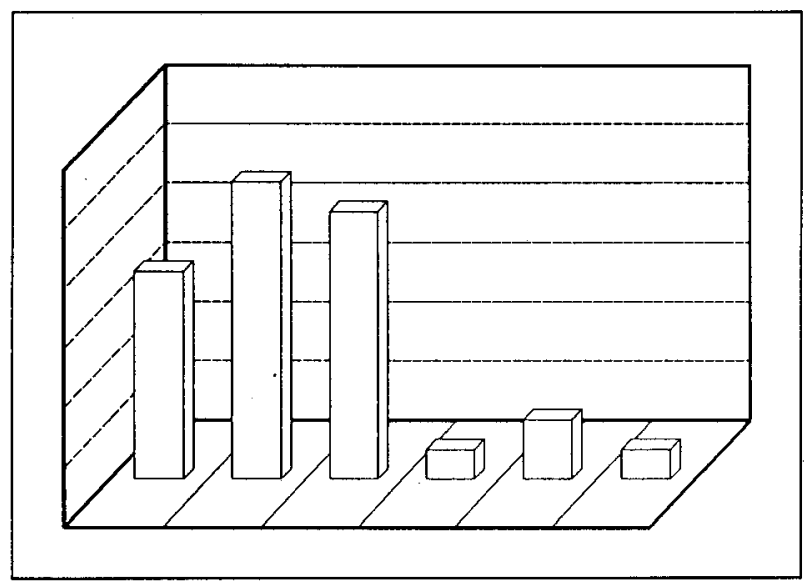

Figura 3. Histograma con la representación de la muestra.

\begin{tabular}{llll}
\multicolumn{2}{l}{ Valores estadísticos } & \multicolumn{2}{l}{ Desviación típica: } \\
Tamaño: & $\mathbf{3 0}$ & Error estandar: & $\mathbf{1 , 3 9 6 6}^{\circ}$ \\
Rango: & $\mathbf{0}^{\circ}-\mathbf{3 4}^{\circ}$ & Límites de & \\
Media: & $\mathbf{1 1 , 0 3 3 3}^{\circ}$ & confianza: & $\mathbf{1 1 , 0 3 3 3}^{\circ}$ \\
Varianza: & $\mathbf{5 8 , 5 1 6 3}$ & & $\mathbf{\pm 4 , 1 8 9 8 ^ { \circ }}$ \\
Coef. Variación: & $\mathbf{6 9 , 3 3 1 6 \%}$ & & $(\mathbf{p}<0,01)$
\end{tabular}

procedentes de indios Pecos del S. XVI, y superiores a las cifras de Kingsley ${ }^{8}$. Los rangos en su límite superior son coincidentes con diferencias de valor sin significación estadística, no así en su límite inferior en el cual llama la atención el valor alcanzado por la retroversión que llega a ser en algunos casos de $-27^{\circ}$ que comparado con nuestros valores mínimos de $0^{\circ}$, representa una notable dispersión. La interpretación de la misma creemos que no corresponde a criterios genotípicos sino más bien a elementos de antropología cultural y tiene que ver con posturas remodelantes tanto al nacimiento como en la edad infantil.

\section{CONCLUSIONES}

$1^{\circ}$ El AAF en hueso seco de adulto en la muestra de población española estudiada tiene un valor medio de $11,03^{\circ} \pm 4,19(\mathrm{p}<0,01)$ con un rango comprendido entre $0^{\circ}$ y $34^{\circ}$.

$2^{\circ}$ Para un $\mathrm{p}<0,01$ los límites de confianza del AAF son de $6,84^{\circ}-15,22^{\circ}$.

\section{AGRADECIMIENTOS}

Al Dr. M. Sanmartín Ruíz por su ayuda en el tratamiento informático de los datos.

\section{BIBLIOGRAFIA}

1. Backman, S. «The proximal end of the femur». Acta Radiol Supl. 145, 1957.

2. Broca. pág. 404, tomo I Loc, Cit. Testut. Tratado de anatomía humana. Editorial Salvat. S.A. Barcelona, 1974.

3. Dega, W. «Anatomy and mechanics of foetal hip. Studied in order to clariticy etiology and pathogenesis of congenital luxation. Cir Organi Mov. Vol. 18, pág. 245, 1933.

4. Durhan, H.A. «Anteversion of the femoral neck in the normal femur». J Am Med Assn 65, pág. 223-224, 1915.

5. Drehman, G.E. «Hip mechanics». J Bone Joint Surg. Vol 41-b, pág. 550, 1959.

6. Esteban Mujica, B. «Desviaciones de los ejes de los miembros en la infancia». Ponencia oficial XIII Congreso Hispano Luso de Cirugía Ortopédica y Traumatología, 1981.

7. Felts, W.J.L. «The prenatal development of the human femur». Amr J Anat. Vol. 94, pág. 1-44, 1954.

8. Kingsley, D.C., and Olmsted, K.L. «A study to determine the angle of anteversion of the neck of the femur». J Bone Joint Surg. Vol. 30-A, e, pág. 745-751, 1948.

9. Lange, F.V., Pitzen, P. «Zur anatomie des oberen femurendes». 2. Orthop. Chir. Vol. 41, pág. 105-134, 1921.

10. Lanz, T. Vom, Wachsmuth, W. Praktische anatomie. Springer Verlag. Berlín, 1938.

11. Le Damany, P. Congenital luxation of the hip. Am J Orthop Surg. 11, 541-567, 1914.

12. Mikulicz, J. Vom, Über individuelle from differenzen an femur an der tibia des menschen. Mit berücksichtiqung der statik des kniegelenks. Arch F Anat Phys Anat Abt. Vol. 1, 351-404, 1874.

13. Olivier, G. Formation du squelette des membres cher l'homme, pag. 131-146 vigat Fréres Edit. Paris 1962.

14. Parsons, F.G. The characters of the English-Thigh-Bone. J Anat and Physiol. Vol. 48, 238-267, 1915.

15. Pearson, K. and Bell, J. A study of the long bones of the English skeleton. Draper's Campany research memoirs. Biometric series 10-11, London Cambridge University Press. 1919

16. Pick, J.W., Stack, J.K. and Anson, B.J. Measurements on the human femur length, diameters and angles. Qurt. Bull. Northwestern Univ. Med. Sch. Vol. 15, 281-290, 1941.

17. Ruff, Ch.B., Hayes, W.C. Cross-sectional geometry of Pecos pueblo femoral and tibial. A biomechanical investigation I: method and general patterns of variation. Am J Phys Anthrop. 60, 359,1983

18. Shand, R. and Steele, M.K. Torsion of the femur. A follow-up report on the case of the Dunlap method for its determination. J Bone Join Surg. Vol. 40-A. 803-816, 1958. 